\title{
Transcriptome of the dead: characterisation of immune genes and marker development from necropsy samples in a free-ranging marine mammal
}

\author{
Joseph I Hoffman ${ }^{1 *}$, Michael AS Thorne ${ }^{2}$, Philip N Trathan ${ }^{2}$ and Jaume Forcada ${ }^{2}$
}

\begin{abstract}
Background: Transcriptomes are powerful resources, providing a window on the expressed portion of the genome that can be generated rapidly and at low cost for virtually any organism. However, because many genes have tissue-specific expression patterns, developing a complete transcriptome usually requires a 'discovery pool' of individuals to be sacrificed in order to harvest mRNA from as many different types of tissue as possible. This hinders transcriptome development in large, charismatic and endangered species, many of which stand the most to gain from such approaches. To circumvent this problem in a model pinniped species, we 454 sequenced cDNA from testis, heart, spleen, intestine, kidney and lung tissues obtained from nine adult male Antarctic fur seals (Arctocephalus gazella) that died of natural causes at Bird Island, South Georgia.
\end{abstract}

Results: After applying stringent quality control criteria based on length and annotation, we obtained 12,397 contigs which, in combination with 454 data previously obtained from skin, gave a total of 23,096 unique contigs. Homology was found to $77.0 \%$ of dog (Canis lupus familiaris) transcripts, suggesting that the combined assembly represents a substantial proportion of this species' transcriptome. Moreover, only $0.5 \%$ of transcripts revealed sequence similarity to bacteria, implying minimal contamination, and the percentage of transcripts involved in cell death was low at 2.6\%. Transcripts with immune-related annotations were almost five-fold enriched relative to skin and represented $13.2 \%$ of all spleen-specific contigs. By reference to the dog, we also identified transcripts revealing homology to five class I, ten class II and three class III genes of the Major Histocompatibility Complex and derived the putative genomic distribution of 17,121 contigs, 2,119 in silico mined microsatellites and 9,382 single nucleotide polymorphisms.

Conclusions: Our findings suggest that transcriptome development based on samples collected post mortem may greatly facilitate genomic studies, not only of marine mammals but also more generally of species that are of conservation concern.

Keywords: Transcriptome, Genomics, Non-model organism, Post mortem, Immune gene, Major Histocompatibility Complex (MHC), Microsatellite, Single Nucleotide Polymorphism (SNP), Marine mammal, Antarctic fur seal, Arctocephalus gazella, Pinniped

\footnotetext{
* Correspondence: joseph.hoffman@uni-bielefeld.de

${ }^{1}$ Department of Animal Behaviour, University of Bielefeld, Postfach 100131, Bielefeld 33501, Germany

Full list of author information is available at the end of the article
} 


\section{Background}

Massively parallel sequencing approaches such as Roche 454 and Illumina HiSeq are transforming the study of non-model organisms by dramatically increasing sequencing depth and coverage in return for a greatly reduced investment in time, labour and resources [1]. This has facilitated the development of transcriptomes, which provide access to the coding sequences of literally thousands of genes and can be mined for genetic markers for use in genome scans, quantitative trait loci mapping and various other applications [2,3].

mRNA sequencing is a particularly powerful approach for developing 'entry level' genomic resources for studying natural populations of non-model organisms, which are often compelling from ecological or evolutionary perspectives but poorly characterised genetically $[4,5]$. The resulting transcriptomes can in turn be mined for Single Nucleotide Polymorphisms (SNPs) which have already proven powerful for gene mapping [6] and are likely to become increasingly important in conservation genetics since they allow the characterisation of population structure and genetic diversity with unprecedented resolution [7]. However, to exhaustively sample a given species' transcriptome usually requires animals to be sacrificed so that transcripts can be harvested from as many different organs as possible [5], with the spleen being a particularly important source of immune genes [8]. Although this is generally considered acceptable for small, tractable and highly abundant organisms such as many insects [9], molluscs [10] and even fish [11], it is less appropriate for species that are large, highly charismatic or threatened. One potential solution, supported by recent expression studies of human cadavers [12] and slaughtered domestic pigs [13], is to sequence tissues obtained shortly after animals have died of natural causes.

Marine mammals provide an interesting case in point, being large and charismatic but extremely difficult to study given that they spend most, if not all, of their time at sea. Unfortunately, many marine mammal populations are also severely depleted due to a combination of historical exploitation [14] and contemporary threats including bycatch and other fisheries interactions [15] and climate change [16]. These factors may explain why only two marine mammal EST libraries, in both cases developed from either skin or blood, have been published to date $[17,18]$. Nevertheless, marine mammals are strong candidates for generating transcriptomes from tissues collected post mortem, since colonially breeding pinnipeds tend to suffer from high mortality rates and occasional mass mortality events $[19,20]$, while many cetacean species are routinely stranded ashore en masse [21].

The Antarctic fur seal (Arctocephalus gazella) is a highly sexually dimorphic pinniped that breeds in crowded rookeries where adult males compete fiercely for access to females [22]. On Bird Island, South Georgia, a colony has been studied since the early 1980s with an aerial walkway providing unprecedented access for tissue sampling and the collection of detailed daily behavioural observations including metre-resolution locations of every adult male sighted ashore [23]. An ongoing molecular study spanning almost two decades has shown that most, if not all, pups are conceived on land [24] and that heterozygosity measured at nine microsatellites correlates with virtually every component of male reproductive success so far analysed including territory holding ability [25], body size [26] and attractiveness to females [27]. However, a paucity of genomic resources for this species, as well as for pinnipeds in general, constrains our ability to understand the underlying mechanisms [28].

To circumvent this problem, we recently developed a partial transcriptome assembly by 454 sequencing nondestructively obtained skin biopsy samples from twelve individuals of this species [29]. In a subsequent pilot study, we showed that it was possible to obtain polymorphic microsatellites targeted towards candidate genes related to immunity and growth by selecting loci that appear variable in silico [30]. We also exploited homology to the dog (Canis lupus familiaris) genome to map transcripts to specific chromosomes, allowing the development of a genome-wide distributed panel of 104 validated, polymorphic SNPs [31].

Although our initial transcriptome was more than adequate for many purposes, immune-related transcripts were not as numerous as originally hoped for, probably due to our having been restricted to the use of skin samples. Moreover, many of the immune-related contigs that we were able to construct had too little depth of sequence coverage to allow SNPs to be called with high confidence. This hampers the development of SNPs within candidate immune genes, a classic example of which, the Major Histocompatibility Complex (MHC), was recently found to be a remarkably strong predictor of survivorship to adulthood in the closely related grey seal, Halichoerus grypus [32].

Here, we 454 sequenced tissues obtained from six different organs (testis, heart, spleen, intestine, kidney and lung) obtained at necropsy from nine adult male fur seals that died of natural causes. We constructed tissuespecific assemblies to compare the representation of immune-related transcripts, including those showing homology to the $\mathrm{MHC}$, and exploited comparative genomics with the dog to map the putative locations of transcripts and in silico derived genetic markers.

\section{Results}

RNA yields from necropsy samples

Yields of total RNA averaged $398 \mathrm{ng} / \mu \mathrm{l}$ per sample and varied significantly by tissue type (Table 1, Two way 
Table 1 Tissue samples collected after death from nine adult male Antarctic fur seals

\begin{tabular}{|c|c|c|c|c|c|c|c|}
\hline \multirow[t]{2}{*}{ Male ID } & \multirow[t]{2}{*}{ Approximate time between death and necropsy (hours) } & \multicolumn{6}{|c|}{ Whole RNA concentration ( $\mathrm{ng} / \mu \mathrm{l})$} \\
\hline & & Testis & Heart & Spleen & Intestine & Kidney & Lung \\
\hline Agaz0820 & 1 & 900 & 160 & 1467 & 105 & 550 & 500 \\
\hline Agaz0821 & 13 & 1888 & 180 & 1100 & 650 & 667 & 160 \\
\hline Agaz1001 & 18 & 578 & 90 & 200 & 34 & 4 & 180 \\
\hline Agaz1006 & 7 & 432 & 160 & 200 & 115 & 175 & 381 \\
\hline Agaz1007 & 8 & 500 & 135 & 389 & 160 & 514 & 160 \\
\hline Agaz1008 & 8 & 1000 & 75 & 375 & 165 & 446 & 85 \\
\hline Agaz1009 & 20 & 938 & 115 & 160 & 200 & 408 & 338 \\
\hline Agaz1010 & 20 & 737 & 120 & 563 & 596 & 534 & 89 \\
\hline Agaz1011 & 30 & - & 64 & 737 & 333 & 175 & 115 \\
\hline
\end{tabular}

A testis sample was unavailable from Agaz1011 due to the carcass having been scavenged by giant petrels (Macronectes giganteus and M. halli).

ANOVA, $F=6.33$, d.f. $=1, P=0.015$ ), with the greatest yields coming from the testis and the lowest from the heart. The time elapsed between death and necropsy did not explain significant variation in RNA concentration (Two way ANOVA, $F=1.09$, d.f. $=1, P=0.302$ ), although weak negative correlations were observed between RNA yield and the time since death for all but one of the tissue types.

\section{Sequence data generated}

The cDNA library was subject to a full 454 sequencing run, yielding 1,046,221 reads of mean length 618bp and totalling 347,354,924bp (see Table 2 for details). Although we obtained only $72.5 \%$ of the total number of reads previously generated by a full 454 run based on skin biopsy samples, read lengths were significantly longer (Figure 1, unpaired $t$-test, $P<2.2 \mathrm{e}^{-16}$ ) due to our having switched from FLX to FLX+ sequencing chemistry.

\section{Transcriptome assemblies}

Assembly of the necropsy data consecutively with Newbler and CAP 3 [33] to minimise redundancy resulted in a total of 14,362 contigs. Selection based on a minimum contig length of $500 \mathrm{bp}$ or, in the case of smaller contigs, for annotation e-values below $1 \mathrm{e}^{-10}$ resulted in 12,397 contigs (86.3\%) being retained for further analysis. Added to these reads was another set of sequencing on skin biopsy samples described previously [29]. These data were assembled using the same bioinformatic pipeline into 20,330 contigs, of which $14,271(70.2 \%)$ were retained after filtering on size and annotation. Besides the assemblies of these two sets of sequences, allowing for direct comparison, a combined transcriptome using both sets of reads was constructed. This comprised 30,765 contigs, of which 23,096 (75.1\%) were retained for the final transcriptome database. Contig lengths were qualitatively very similar among the three assemblies (Figure 1) and the combined assembly had an average contig length of $971 \mathrm{bp}$.

To explore the differential representation of transcripts among the three assemblies, we BLASTed the combined assembly against those derived from necropsy and skin. A highly stringent e-value threshold of $1 \mathrm{e}^{-100}$ was employed to ensure that any matches are indicative of near-perfect similarity. As expected, the majority of contigs from the combined assembly revealed strong homology to contigs from the necropsy $(n=11,327$, $49.0 \%)$ and skin $(n=12,897,55.8 \%)$ assemblies, with the total number of overlapping contigs from all three sets being 19,869 ( $86.0 \%$ of the combined set). However, only 4,355 of the combined assembly contigs (18.9\%) matched both necropsy and skin, implying a limited degree of overlap between the latter two assemblies (Figure 2).

\section{Functional annotation}

Sequence similarity searches to the non-redundant (nr) database produced matches for a total of 15,073 contig sequences $(65.3 \%$ of the total 23,096 contigs). The

Table 2 Summary of $\mathbf{4 5 4}$ sequence data obtained from the Antarctic fur seal

\begin{tabular}{llllllll}
\hline & Testis & Heart & Spleen & Intestine & Kidney & Lung & Skin \\
\hline Number of reads & 97,799 & 38,332 & 275,366 & 167,555 & 336,223 & 130,946 & $1,443,397$ \\
Mean read length & 823 & 689 & 597 & 567 & 546 & 740 & 542 \\
Total base pairs (bp) & $33,342,585$ & $12,996,565$ & $90,644,969$ & $57,138,133$ & $108,713,181$ & $44,519,491$ & $412,933,200$ \\
\hline
\end{tabular}

Results are given separately for each type of tissue obtained at necropsy as well as for a normalised cDNA library previously generated from skin biopsy samples [29]. 


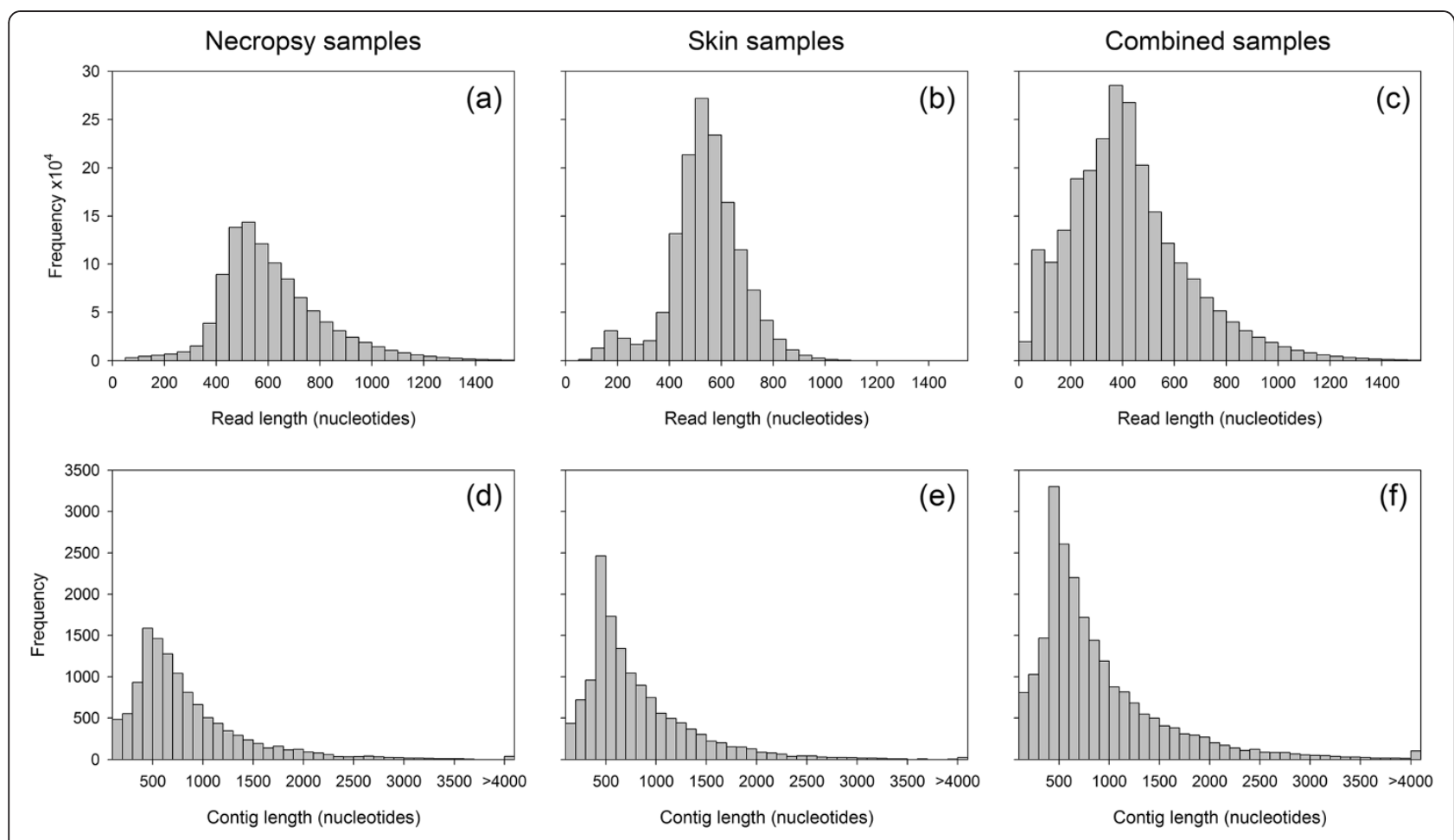

Figure 1 Read $(a-c)$ and contig (d-f) length distributions for the necropsy (a and d), skin (b and e) and combined (c and f) libraries.

majority of sequences showed top matches to eukaryotic species, with the remaining matches involving mostly pathogenic bacteria (see Figure 3a-c). Reassuringly, the proportion of bacterial matches was actually lower in the necropsy assembly than in the skin assembly $(0.5 \%$ versus $2.1 \%$ ), suggesting that we managed to avoid contaminating the samples during the necropsy procedure. Figure $3 \mathrm{~d}-\mathrm{f}$ shows the taxonomic breakdown of the top matches obtained for the three assemblies by species. Although there was minor variation among the assemblies, the top ten matches were consistently to mammalian species, with the giant panda (Ailuropoda melanoleuca) and the dog being the two most frequently represented.

Using standard GO annotation, contigs from the combined assembly were next classified according to three major functional categories: biological process, molecular function and cellular component. Molecular function made up the majority of GO designations $(n=53,189)$ followed by biological processes $(n=46,947)$ and then cellular component $(n=32,140)$. These data are summarised in Figure 4, which reveals a functionally diverse range of

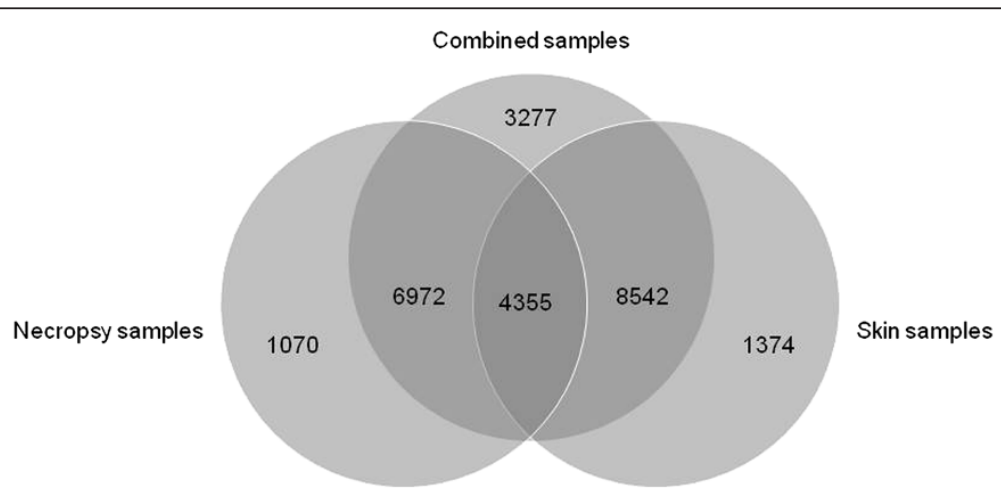

Figure 2 Venn diagram showing the differential representation of transcripts among the necropsy, skin and combined assemblies (see Results for details). The numbers of contigs represented within each portion of the diagram are shown. Light grey portions correspond to contigs observed within a single assembly, mid grey portions to contigs common to two different assemblies, and the dark grey portion corresponds to contigs shared among all three assemblies. 
Necropsy assembly

(a)

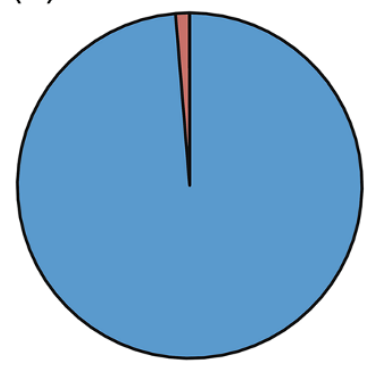

(d)

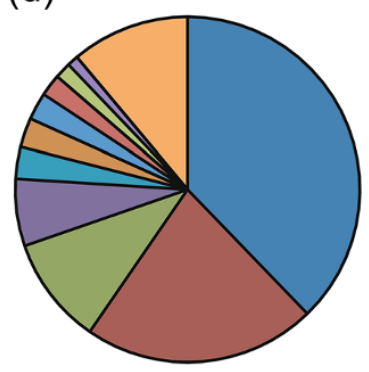

Skin assembly

(b)

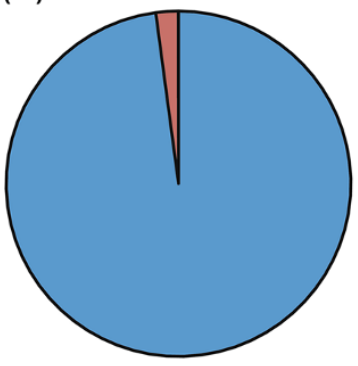

(e)

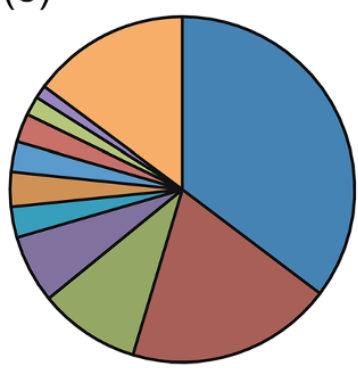

Combined assembly

(c)

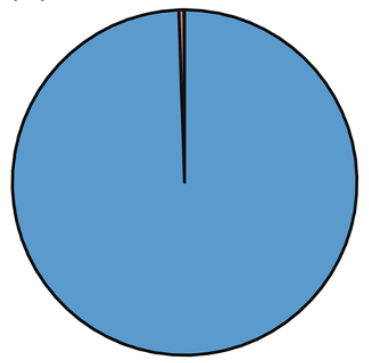

- Eukaryotes

Bacteria

पViruses and viroids

(f)

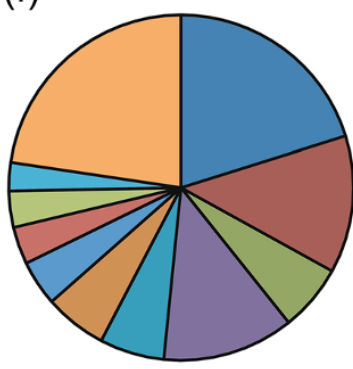

-Ailuropodamelanoleuca

$\square$ Canis lupus familiaris

-Mustela putorius furo

ㅁHomo sapiens

口Equus caballus

口Bostaurus

口Sus scrofa

口Mus musculus

$\square$ Macaca mulatta

$\square$ Loxodonta africana

$\square$ Pan troglodytes

口Other

Figure 3 Taxonomic distribution of top BLAST hits for the necropsy ( $a$ and d), skin (b and e) and combined (c and f) assemblies, classified into eukaryotes, bacteria or viruses $(a-c)$ and according to the top ten species represented (d-f).

annotation terms. Summaries generated separately for the necropsy and skin assemblies were qualitatively very similar and are therefore not shown.

To discount the possibility that the necropsy assembly could be dominated by genes involved in cell death, we next interrogated the three assemblies for contigs with GO-codes relating to apoptosis. Figure $5 \mathrm{a}$ shows that, although contigs representing apoptosis-related genes are almost twice as numerous in the necropsy assembly than in the skin assembly, their overall percentages are low in all three assemblies. Furthermore, relatively little variation was found in the numbers and percentages of apoptosis-related transcripts among the different types of tissue obtained at necropsy.

We next exploited GO annotation terms to extract a total of 521 immune-related contigs, which are listed in Additional file 1: Table S1. These had a mean length of $854 \mathrm{bp}$ and contained a total of 1,471 SNPs (see subsequent section for SNP discovery). Associated GO terms were diverse, including 'immune system process', 'immune response', chemokine activity', 'antigen processing and presentation', and 'T cell selection'. As anticipated, immune-related contigs were over three times more numerous in the necropsy assembly than in the skin assembly, despite the overall amount of 454 sequence data being lower (Figure $5 \mathrm{~b}$ ). This is largely attributable to our having included spleen tissues in the necropsy assembly, which alone yielded 433 contigs with GO annotations relating to immunity, accounting for $13.2 \%$ of all spleen-specific contigs. Moreover, in contrast to the other tissues, four of the top ten most abundant transcripts identified in the spleen were immune related (Additional file 2: Table S2), implying relatively high levels of expression.

\section{Comparative genomics between the fur seal and dog}

To shed light on the genomic distribution of the 454 sequence data, we mapped unassembled reads and contig sequences to the dog genome. A total of 1,521,212 individual reads (61.1\%) successfully mapped, with $50.1 \%$ of these uniquely mapping to a single chromosome. A strong positive relationship was found between the number of reads mapping to a given chromosome and the length of that chromosome in the $\operatorname{dog}\left(\mathrm{r}^{2}=0.73, n=39, P<0.0001\right)$, indicating a fairly even genomic distribution. As anticipated, the number of contigs from the combined assembly mapping to the dog genome was highly dependent on the e-value threshold applied in the BLAST analysis. This 


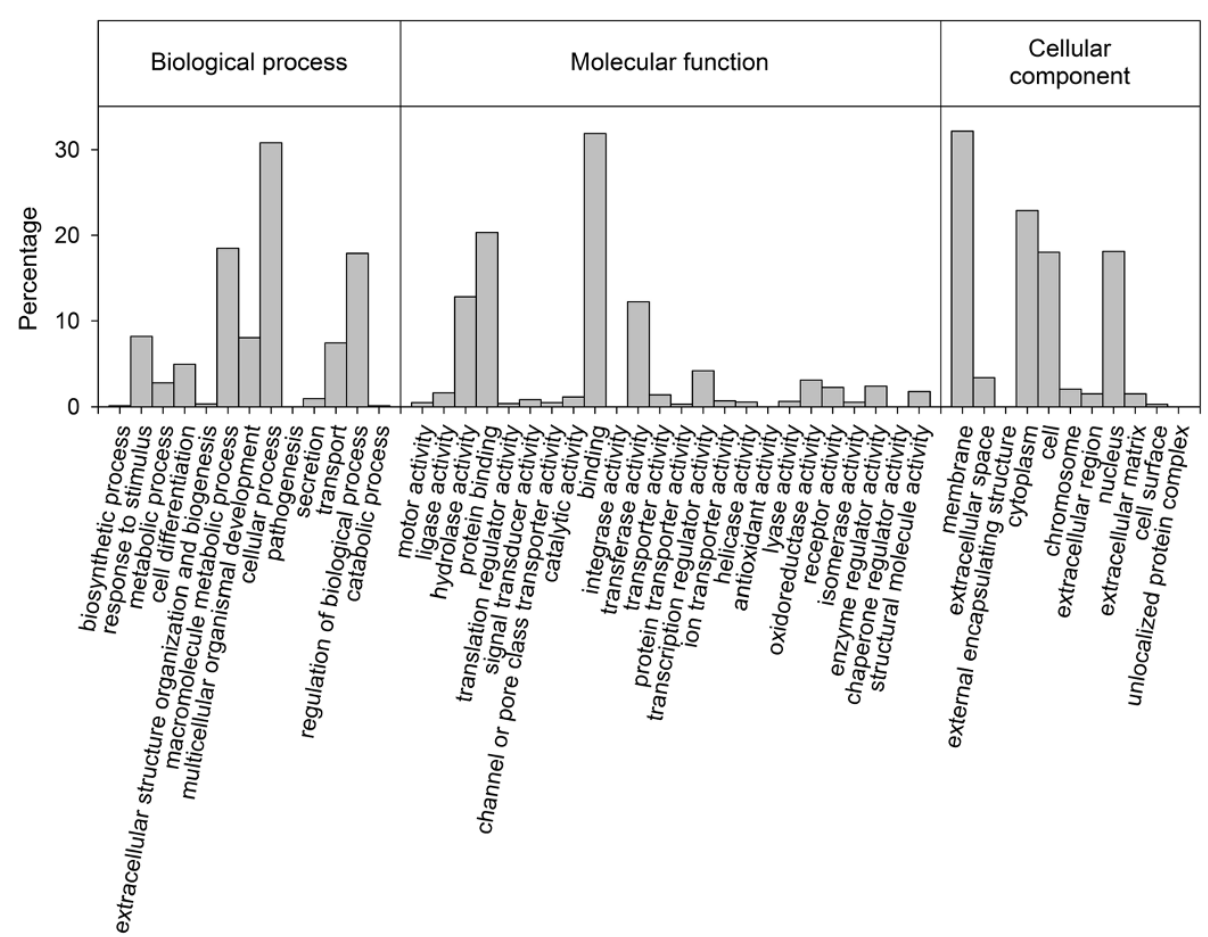

Figure 4 Gene ontology classifications for the combined assembly, summarized for three major categories: biological process, molecular function and cellular component.

varied from 22,424 (97.1\%) with a threshold of $1 \mathrm{e}^{-4}$ through $17,121(74.1 \%)$ with a threshold of $1 \mathrm{e}^{-50}$ to 4,118 $(17.8 \%)$ with a threshold of 0 . The proportion of contigs mapping to more than one chromosome also decreased with increasing stringency, $(50.8 \%, 77.6 \%$ and $89.9 \%$ for $1 \mathrm{e}^{-4}, 1 \mathrm{e}^{-50}$ and 0 respectively). To provide an optimal balance between the number of mapped contigs and the stringency required for uniqueness, we therefore chose an e-value of $1 \mathrm{e}^{-50}$ for all subsequent analyses when mapping between fur seal contigs and the dog genome. Using these data, we obtained a similar pattern to the reads, in which the number of mappings correlated positively with chromosome length in the $\operatorname{dog}\left(\mathrm{r}^{2}=0.78\right.$, $n=39, P<0.0001)$. The detailed genomic distribution of contigs inferred by mapping to the dog is shown in Figure 6.

Another question that can be addressed through comparative genomics is the extent to which a given collection of transcripts represents an exhaustive transcriptome. To evaluate the completeness of the fur seal transcriptome, we therefore explored homology between the combined assembly and the complete set of dog transcripts using a standard e-value threshold of $1 \mathrm{e}^{-10}$. A total of $14,435(62.5 \%)$ fur seal contigs showed homology to 19,691 of the 25,559 (77.0\%) dog transcripts, suggesting that the combined assembly represents a substantial proportion of the fur seal transcriptome.
We also exploited homology between the fur seal and dog to explore the tissue-specific representation of transcripts revealing homology to 21 different canine MHC genes. The mapping data were consistent with the expression of five class I genes, ten class II genes and three class III genes (Table 3). The number of reads per million mapping to these genes varied significantly by MHC class (Two way ANOVA; $F=6.16$, d.f. $=1$, $P=0.014$ ) but not by tissue (Two way ANOVA; $F=0.13$, d.f. $=1, P=0.723$ ). Nevertheless, total number of reads per million was highest for class I and II genes in the spleen, whereas for class III genes this was highest in the lung. The skin also had by far the lowest representation of MHC-homologous transcripts, with only 663 reads per million in comparison to 8330 reads per million for spleen.

\section{Molecular marker discovery}

Interrogating the combined assembly for perfect microsatellites with at least five repeat units identified 2,592 loci, 1,895 (73.1\%) of which comprised dinucleotides, $561(21.6 \%)$ trinucleotides and $92(3.5 \%)$ tetranucleotides. These were located within 2131 different contigs, of which 1,185 (55.6\%) were functionally annotated with respect to the $\mathrm{nr}$ database and $1,765(82.8 \%)$ revealed significant matches to locations within the dog genome. To allow direct comparison with results previously obtained from the skin transcriptome assembly [29], we 

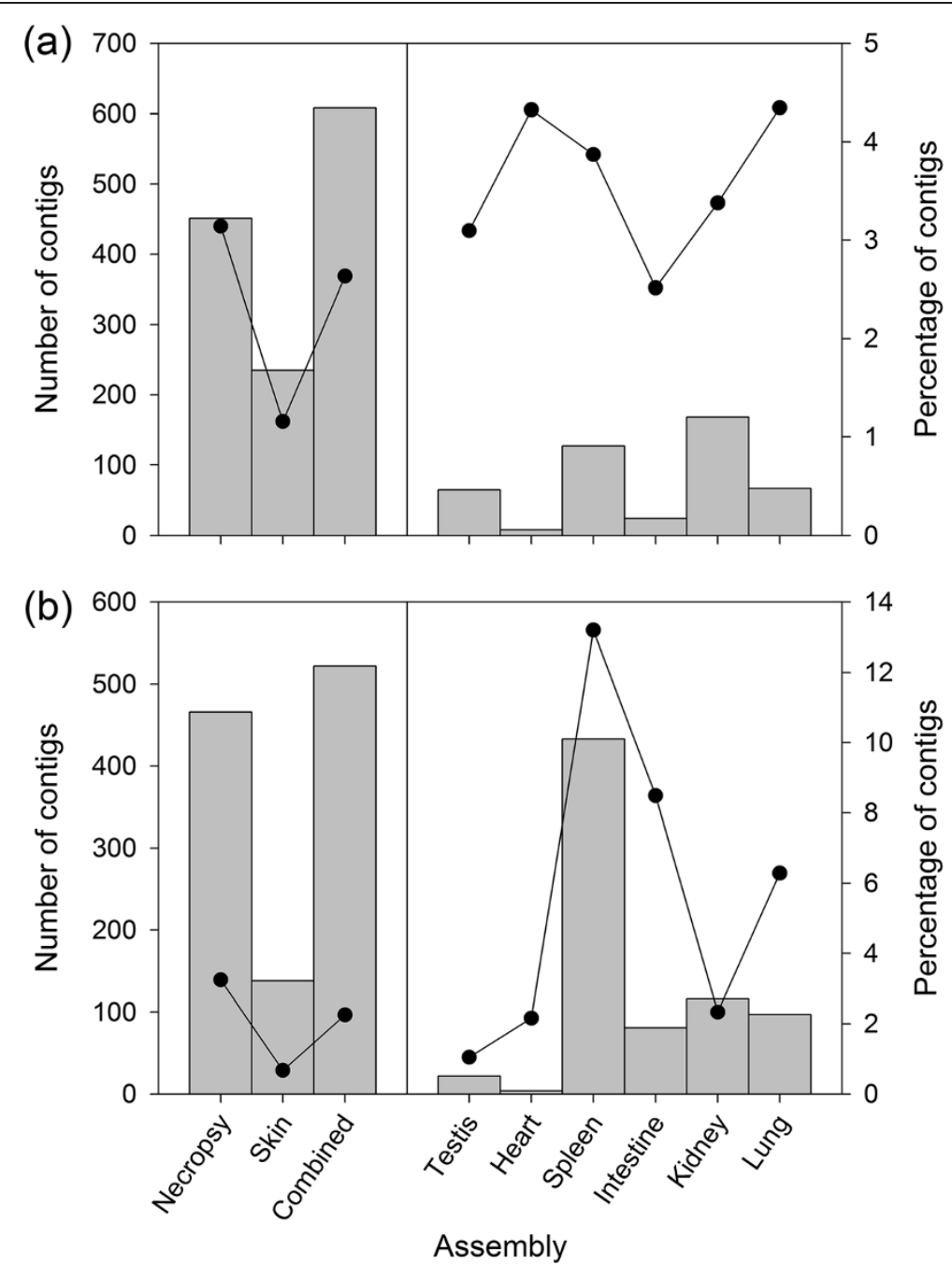

Figure 5 Numbers and percentages of contigs from each assembly, denoted by vertical bars and points respectively, with GO annotations relating to (a) apoptosis; and (b) immunity.

carried out an initial round of SNP discovery using the program SWAP454. Applying a 'strict' criterion in which SNPs were accepted when at least $33 \%$ of the reads differed from the reference sequence and the minor allele was observed at least eight times, a total of 1,585 SNPs were identified. Applying a 'relaxed' criterion that required only $10 \%$ of the reads to differ from the reference sequence and two copies of the minor allele to be present, the number of SNPs increased to 11,454. Next, to circumvent dependency on mapping the raw sequence reads to a reference assembly, we used the Newbler mapping program, identifying a total of 14,574 SNPs with high confidence. These were located within 5,205 different contigs, of which 3,617 (69.5\%) were functionally annotated with respect to the $\mathrm{nr}$ database and 4,028 (77.4\%) revealed significant matches to locations within the dog genome. To explore the corresponding SNP parameter space, we fitted a local regression to the raw data. Figure 7 reveals a clear peak in the number of SNPs identified at a MAF of around 0.3 and a $\log$ depth of coverage of approximately 1.2 , which corresponds to a respectable 16x depth of coverage. The inferred genome-wide distributions of microsatellites and SNPs identified by the Newbler mapping program are shown in Figure 6.

\section{Discussion}

Transcriptome sequencing has become an increasingly popular means of developing genomic resources for nonmodel organisms. However, to sample a transcriptome exhaustively usually requires animals to be sacrificed so that mRNA can be harvested from multiple tissues. To circumvent this problem in a natural population of Antarctic fur seals, we 454 sequenced cDNA from various tissues obtained at necropsy from nine adult males that died of natural causes. This appears to have been 


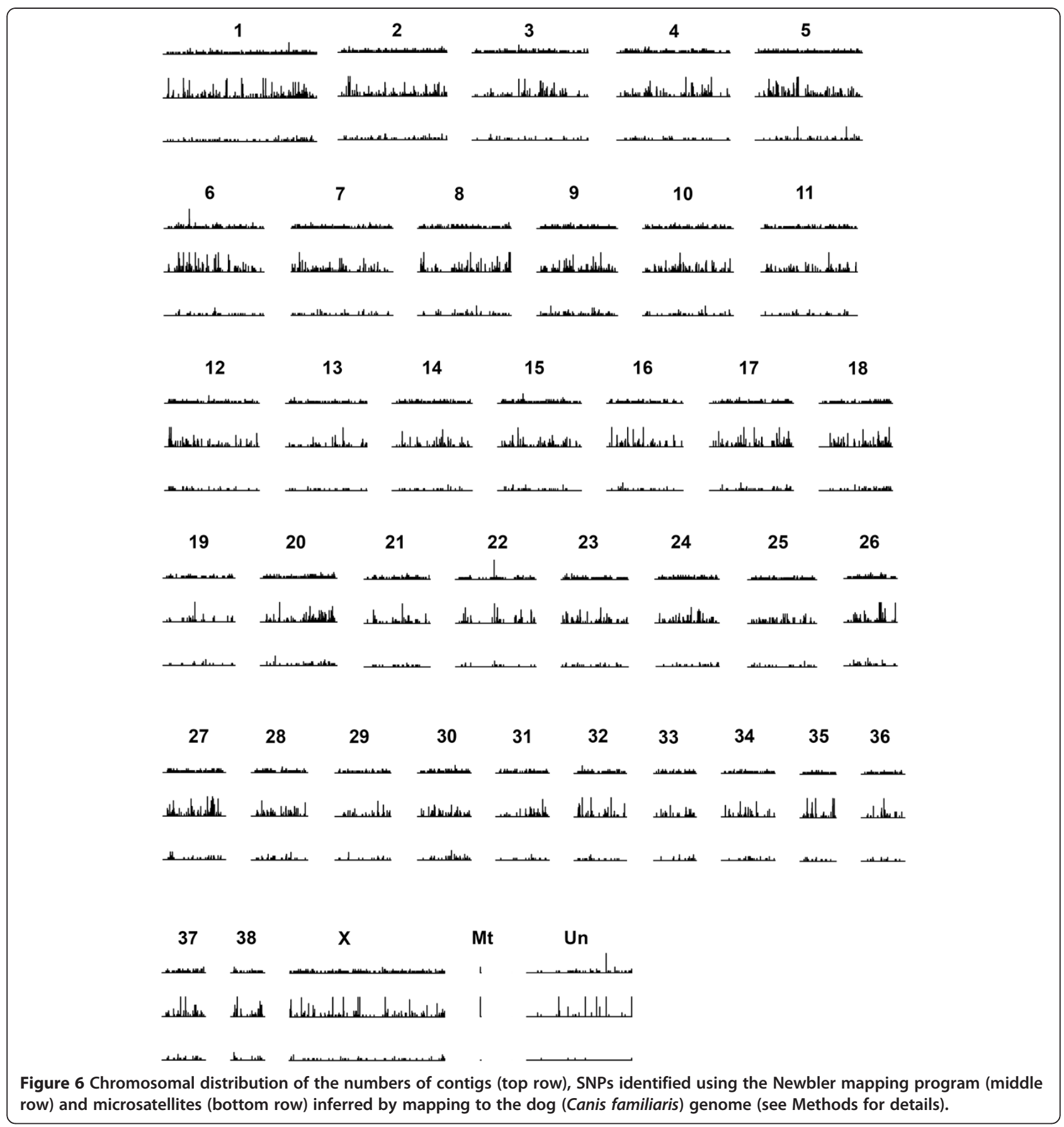

remarkably successful, leading to measurable increases in the number and diversity of transcripts characterised, a greater representation of transcripts involved in immunity, and many more genetic markers discovered.

It is important to acknowledge that this study is not the first to apply high throughput sequencing to tissues obtained after death. For example, Cherel et al. [13] recently explored links between gene expression and meat quality traits in recently slaughtered pigs, and Kang et al. [12] characterised the developmental progression of gene expression patterns in the human brain. However, such studies are few and far between, and tend to focus on humans and their companion species. Our study is novel in that we have harvested multiple organs from animals dying of natural causes in a natural population of a nonmodel organism in order to generate an exhaustive as possible de novo transcriptome assembly.

Given that some of our samples were obtained from adult males that had died up to thirty hours prior to necropsy (Table 1), we initially held concerns over the 
Table 3 Numbers of reads per million mapping to known canine MHC genes, summarised for the various tissues plus the combined assembly (see Methods for details)

\begin{tabular}{|c|c|c|c|c|c|c|c|c|c|c|c|}
\hline \multirow[t]{2}{*}{ Class } & \multirow[t]{2}{*}{ Gene } & \multirow[t]{2}{*}{ Reference } & \multirow{2}{*}{$\begin{array}{l}\text { Chromosome } \\
\text { in dog }\end{array}$} & \multicolumn{7}{|c|}{ Tissue } & \multirow[t]{2}{*}{ Combinec } \\
\hline & & & & Heart & Intestine & Kidney & Lung & Skin & Spleen & Testis & \\
\hline | & DLA-64 & ENSCAFG00000000500 & 12 & 78.4 & 257.1 & 324.8 & 390.2 & 85.2 & 687.6 & 61.5 & 240.7 \\
\hline । & DLA-79 & ENSCAFG00000008234 & 18 & 0.0 & 0.0 & 14.9 & 0.0 & 15.6 & 3.6 & 0.0 & 10.5 \\
\hline | & DLA-88 Precursor & ENSCAFG00000000487 & 12 & 0.0 & 269.1 & 268.2 & 451.5 & 43.6 & 654.8 & 61.5 & 210.3 \\
\hline । & DLA-88 & ENSCAFG00000000492 & 12 & 78.4 & 191.4 & 283.1 & 550.9 & 1.0 & 753.0 & 71.7 & 207.8 \\
\hline । & Tapasin & ENSCAFG00000000972 & 12 & 0.0 & 41.9 & 110.2 & 91.8 & 2.1 & 149.2 & 30.7 & 50.8 \\
\hline$\|$ & BTNL2 & ENSCAFG00000000797 & 12 & 0.0 & 0.0 & 0.0 & 0.0 & 0.0 & 0.0 & 0.0 & 0.0 \\
\hline$\|$ & CD74 & ENSCAFG00000018101 & 4 & 52.3 & 71.8 & 143.0 & 145.4 & 0.0 & 338.3 & 41.0 & 88.7 \\
\hline$\|$ & DLA-DRA & ENSCAFG00000000803 & 12 & 26.1 & 59.8 & 134.1 & 298.4 & 165.2 & 516.6 & 20.5 & 198.3 \\
\hline$\|$ & DQ Alpha precursor & ENSCAFG00000000812 & 12 & 0.0 & 6.0 & 65.6 & 168.3 & 83.1 & 247.4 & 10.2 & 96.7 \\
\hline$\|$ & HLA-DMA & ENSCAFG00000000848 & 12 & 0.0 & 0.0 & 3.0 & 0.0 & 1.0 & 7.3 & 0.0 & 2.0 \\
\hline$\|$ & HLA-DOA & ENSCAFG00000000896 & 12 & 0.0 & 0.0 & 0.0 & 0.0 & 0.0 & 3.6 & 20.5 & 1.5 \\
\hline$\|$ & HLA-DOB & ENSCAFG00000000819 & 12 & 0.0 & 0.0 & 0.0 & 0.0 & 0.0 & 0.0 & 0.0 & 0.0 \\
\hline$\|$ & HLA-DPB1 & ENSCAFG00000000900 & 12 & 0.0 & 0.0 & 3.0 & 0.0 & 3.1 & 0.0 & 0.0 & 2.0 \\
\hline$\|$ & HLA-DQB1 & ENSCAFG00000000814 & 12 & 0.0 & 17.9 & 26.8 & 53.6 & 6.2 & 54.6 & 10.2 & 20.4 \\
\hline$\|$ & HLA-DRB1 & ENSCAFG00000000806 & 12 & 52.3 & 59.8 & 83.4 & 336.7 & 40.5 & 232.8 & 51.2 & 95.7 \\
\hline$\|$ & IK cytokine & ENSCAFG00000005869 & 2 & 52.3 & 6.0 & 62.6 & 38.3 & 128.9 & 36.4 & 61.5 & 84.2 \\
\hline$\|$ & RFX3 & ENSCAFG00000002053 & 1 & 0.0 & 47.8 & 8.9 & 0.0 & 0.0 & 0.0 & 61.5 & 8.5 \\
\hline III & BAG6 & ENSCAFG00000000575 & 12 & 130.7 & 131.6 & 122.2 & 122.4 & 4.2 & 80.0 & 706.9 & 89.2 \\
\hline III & HSP70 & ENSCAFG00000000641 & 12 & 2117.7 & 998.7 & 2413.4 & 7789.6 & 83.1 & 4565.6 & 624.9 & 1730.3 \\
\hline III & LTA & ENSCAFG00000000515 & 12 & 0.0 & 0.0 & 0.0 & 0.0 & 0.0 & 0.0 & 0.0 & 0.0 \\
\hline \multirow[t]{5}{*}{ III } & TNF & ENSCAFG00000000517 & 12 & 0.0 & 0.0 & 0.0 & 7.7 & 0.0 & 0.0 & 0.0 & 0.5 \\
\hline & & & Total class I & 156.9 & 759.5 & 1001.1 & 1484.5 & 147.6 & 2248.2 & 225.4 & 720.1 \\
\hline & & & Total class II & 183.0 & 269.1 & 530.4 & 1040.7 & 428.1 & 1437.0 & 276.6 & 598.0 \\
\hline & & & Total class III & 2248.4 & 1130.2 & 2535.6 & 7919.7 & 87.3 & 4645.6 & 1331.9 & 1820.0 \\
\hline & & & Grand total & 2588.3 & 2158.8 & 4067.1 & 10444.8 & 663.0 & 8330.8 & 1833.9 & 3138.1 \\
\hline
\end{tabular}

quantity of the RNA that could be extracted and whether this could have downstream impacts on the amount of resulting 454 sequence data. As anticipated, significant variation was found in the amount of total RNA that could be extracted from the different tissues, the more fibrous heart yielding the lowest average concentration despite our having used a bead mill. However, there was no clear relationship between RNA yield and the time elapsed between death and necropsy. Moreover, 454 read lengths were actually longer on average than previously obtained for the skin transcriptome. Although this is almost certainly due to our having switched from FLX to FLX+ sequencing chemistry, it nevertheless appears that using RNA from recently dead animals did not have a major detrimental impact on sequence length.

An important caveat to the above is that organs were harvested shortly after death (1-30 hours, mean $=13.9$ hours) in order to minimise the risk of RNA degradation. Moreover, the prevailing climate at Bird Island during the austral summer is cool (usually between 1.5 and 4.5 degrees centigrade). It remains unclear how much highquality RNA will be retained in carcasses at more advanced stages of decomposition, nor how dependent RNA degradation will be upon ambient temperature, making it difficult to extrapolate to other study systems. To evaluate this in fur seals would require significant further effort, since carcasses would need to be repeatedly sampled at different time intervals, generating multiple cDNA pools for sequencing. Moreover, such an experiment would ideally evaluate the importance of factors such as carcass size and prevailing environmental conditions.

For the previous assembly based on skin samples [29], we employed the cDNA option within Roche Newbler assembler version 2.3. However, a recent paper comparing the merits of five different assemblers found that this program performed poorly, generating the assembly with the greatest sequence redundancy and lowest proportion of mappings to reference sequences [34]. In contrast, a prerelease version of Newbler 2.5 was identified as the joint top performing assembler, with further improvements 


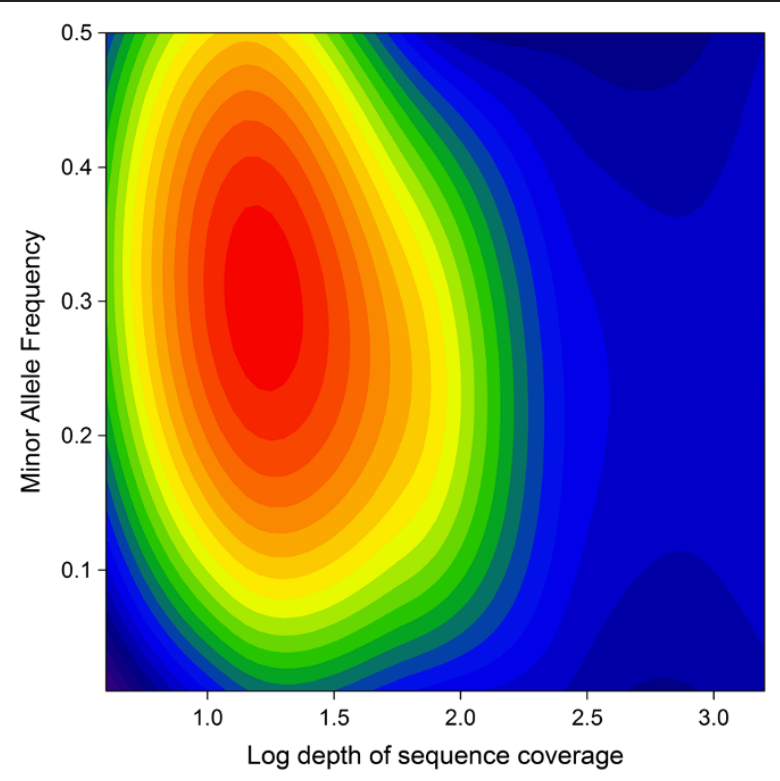

Figure 7 Variation in the number of SNPs discovered in the combined assembly with Minor Allele Frequency (MAF) and depth of sequence coverage. Local regression was used to fit a smoothed spline to the raw dataset, comprising 14,574 SNPs identified using the Newbler mapping program, implemented using the 'locfit' package in $\mathrm{R}$. The area shaded in red indicates the parameter space associated with the greatest numbers of SNPS discovered.

being obtained through the concurrent use of more than one assembler. To obtain the best possible quality transcriptome, we therefore assembled our data consecutively with Newbler 2.5 and CAP 3. After filtering on the basis of size and annotation, we obtained 23,096 contigs, which is considerably more than the equivalent value of 14,271 obtained when the original skin data were re-assembled and filtered based on the same criteria. Taken together with the limited degree of overlap between the necropsy and skin assemblies, this suggests that our improved transcriptome is larger and contains numerous transcripts that had not previously been described. This is consistent with previous studies that have found tissue-specific patterns of gene expression [35,36].

Quality filtering for size and annotation led to many small contigs being discarded from the final assembly. However, this was reflected in a greatly improved rate of annotation $(65.3 \%$ of contigs yielded BLAST hits with an e-value cutoff of $1 \mathrm{e}^{-10}$ ) relative to the original skin transcriptome $(47.0 \%$ of contigs yielded BLAST hits with an e-value cutoff of $1 \mathrm{e}^{-4}$ ). As found previously, the majority of matches were to vertebrates, with the giant panda and the dog being the two most frequently represented species. Interestingly, more matches were obtained to the panda than the dog for the necropsy and skin assemblies, but this pattern was reversed for the combined assembly. This difference may reflect the fact that the combined assembly contains substantially more larger transcripts than either the necropsy or skin assemblies (number of transcripts $>1,000 \mathrm{bp}$ in length $=7,780,3,789$ and 4,320 respectively) due to the greater total number of reads available.

Reassuringly, the proportion of BLAST matches to bacterial sequences was actually lower for the necropsy than the skin assembly ( $0.5 \%$ versus $2.1 \%)$. This not only implies that we managed to avoid contaminating the samples during the necropsy procedure, but may also indicate that the seals we necropsied were not suffering from heavy bacterial infections at the time of death. The difference between the two assemblies if genuine would also be consistent with a previous study that isolated a diverse bacterial assemblage from fight wounds and lesions in adult males of this species [37]. Thus, the greater representation of bacterial sequences in skin could quite possibly reflect the relatively unsanitary conditions of life in a crowded fur seal breeding colony.

Although it was not critical that our transcriptome be perfectly representative of normal patterns of gene expression, we initially held concerns that a transcriptome derived from necropsy samples could be dominated by transcripts involved in cell death. To evaluate this possibility, we therefore interrogated each of the assemblies, including the tissue-specific assemblies, for GO terms relating to apoptosis. Contigs with apoptosis-related GO terms were around twice as numerous in the necropsy than in the skin assembly. However, their overall percentage was low, at only $2.6 \%$ for the combined assembly and rising to a maximum of $4.3 \%$ for the heart and lung tissues. Thus, although our data are consistent with a certain degree of up-regulation of genes involved in apoptosis, there is no suggestion of a major bias being present.

This study was partly motivated by the limited representation of immune-related transcripts in our original skin assembly, together with the fact that many of these had insufficient depth of sequence coverage to allow SNPs to be identified with high confidence. Using the same approach as above, but this time filtering for transcripts based on GO annotations relating to immunity, we identified a total of 521 immune-related contigs, almost four times more than in the skin assembly. This improvement appears largely attributable to the inclusion of samples from the spleen, which carried by far the greatest proportion of immune-related transcripts, at $13.2 \%$. However, the main causes of death in adult male Antarctic fur seals are fight wounds and pneumonia, raising the possibility that at least some of these individuals could have been mounting an immune response to bacterial infection prior to death. This is difficult to judge given the low proportion of sequence matches to bacteria, but could potentially have contributed towards 
the elevated representation of immune-related transcripts in the necropsy assembly.

Through comparative genomics with the dog, we also analysed the tissue-specific representation of transcripts revealing homology to 21 different canine MHC genes. All but three of these genes, including class I, II and III genes, were represented in at least one tissue. Consistent with immune-related contigs being four times more common in the spleen, the total number of transcripts per million mapping to MHC class I and II genes was also highest for the spleen, followed by the lung. A contrasting pattern was obtained for the MHC class III, with the lung having almost twice as many transcripts per million mapping as the spleen. This may reflect the role of class III genes in mounting the immune response via innate immunity, inflammation and immunomodulation [38], processes that could conceivably be of greater importance in sensitive mucosal tissues such as the lung. Overall, we also found that MHC-related transcipts had the lowest representation in the skin, providing further justification for our having expanded and improved upon our original transcriptome.

Despite seals and dogs having diverged approximately 43 million years ago [39], the two genomes appear to have retained large syntenic blocks [40]. Sequence homology is also strong enough to allow the flanking sequences of most pinniped microsatellites to be mapped to unique locations in the dog $[28,40]$, making the canine genome a powerful resource for comparative purposes. We therefore took previous seal studies a step further by inferring the genomic distribution of $1,521,212$ reads (61.1\%), 17,121 contigs (74.1\%), 2,119 microsatellite loci (81.8\%) and 9,382 SNPs (58.0\%) by reference to the dog. Much as expected, the resulting distributions appear relatively even (Figure 6), as supported by a strong positive correlation between the number of contigs mapping to a given chromosome and the length of that chromosome in the dog. Moreover, although the mapping locations should be regarded as putative, we have reasons to believe that a substantial proportion will be correct. For example, Hoffman et al. [28] previously found that four microsatellite loci, described as putatively X-linked because they were homozygous in 84 males but carried the expected proportions of heterozygote genotypes in females, all mapped to the $\mathrm{X}$ chromosome in the dog [28]. Similarly, a SNP recently developed within a transcript revealing homology to mitochondrial NADH dehydrogenase revealed a pattern in which all individuals were called as homozygotes but for different alleles [31]. Nevertheless, to shed further light on synteny between the two genomes, it would be desirable to develop a high-density comparative linkage map. This is made feasible for the first time by the large number of genetic markers we have identified in this study (see below).
Mining the combined transcriptome assembly for microsatellites yielded marginally more markers than found in the original skin assembly (2,592 loci versus 2,271 loci respectively), although these numbers are not strictly comparable because different programs were used. A more obvious improvement was observed for SNPs, with SWAP454 identifying 2.5 times as many SNPs at the 'strict' level $(1,585$ versus 642$)$ and 1.8 times more SNPs at the 'relaxed' level $(11,454$ versus 6,261$)$. This is perhaps to be expected given the increased number and diversity of contigs and the improved depth of coverage, to which SNP discovery is particularly sensitive. Even more SNPs were identified by the Newbler mapping program $(n=14,574)$, with a clear peak in the parameter space corresponding to a MAF of around 0.3 and a depth of coverage of approximately $16 x$. This suggests that many of these SNPs may comfortably exceed the minimum selection criteria of at least three nonduplicate reads showing the variant and seven or more high-quality reads in total. One possible reason for the difference in the number of SNPs called by the two programs could be that SWAP454 relies on mapping the raw sequence reads back to a reference sequence, in this case the transcriptome assembly. This can lead to some loss of data due to incomplete mapping. Moreover, because the program only calls SNPs on the basis of reads that map reliably to a single contig, redundancy within the assembly, whether unintentional or due to the use of assembly methods that classify related contigs into 'isogroups' as constructed using the Newbler assembler, could potentially reduce the total number of SNPs called.

\section{Conclusions}

By 454 sequencing samples obtained at necropsy, we have developed a greatly improved transcriptome assembly, thereby facilitating future evolutionary genetic studies of an important pinniped species, the Antarctic fur seal. We have also demonstrated that post mortem sampling provides a viable alternative to sacrificing animals, with positive implications for developing transcriptomes for charismatic and / or threatened species.

\section{Methods}

\section{Animal sampling}

Tissue samples were collected from nine freshly dead adult male Antarctic fur seals at Freshwater Inlet on Bird Island, South Georgia (54 $04^{\circ} \mathrm{S}, 38^{\circ} 02^{\prime} \mathrm{W}$ ) during the austral summer of 2010 / 2011. All specimens were known to have died within the last 30 hours by direct observation, and were transported immediately to the station laboratory to prevent scavenging by seabirds. The necropsies were conducted systematically within the shortest possible time window after death (see 
Table 1 for details). Tissue samples from all six organs were available for all but one of the animals, Agaz1011, due to this individual's testes having been scavenged by giant petrels (Macronectes giganteus and M. halli). Sampling equipment was sterilised using 95\% ethanol between uses. Samples were transferred to RNAlater ${ }^{\circledR}$ and stored individually at $-20^{\circ} \mathrm{C}$ for up to one month before being placed in a $-80^{\circ} \mathrm{C}$ freezer for transport back to the UK.

\section{RNA isolation and cDNA generation}

Approximately $10 \mathrm{mg}$ of each tissue sample was disrupted and homogenised by bead milling within a TissueLyserII (Qiagen). Total RNA was then extracted using a Qiagen RNeasy ${ }^{\circledR}$ mini kit following the manufacturer's recommended protocols, with an optional on-column DNAse digestion step included. The resulting RNA pellets were resuspended in $50 \mu \mathrm{l}$ of RNAse-free water (Ambion) and quantified using PicoGreen (Invitrogen) fluorometry. RNA quantity and quality were also assessed visually by running a fraction of each isolate on a 1\% agarose gel. Total RNA samples were pooled in equimolar ratios, as far as possible, for each of the tissue types. PolyA+ RNA was purified from total RNA using selection on Oligo-dT containing paramagnetic beads from the MicroPoly(A) PuristTM mRNA Purification Kit (Ambion, Life Technologies) according to the manufacturer's instructions. 200ng mRNA of each tissue was used for cDNA Rapid Library construction according to 454/Roche FLX+ protocols. The individual tissue libraries were MID tagged, pooled and sequenced on a Roche Genome Sequencer FLX+ instrument using the GS FLX Titanium Sequencing Kit XL+ (Roche).

\section{Skin transcriptome data}

Sequence data were also available from a previously published skin transcriptome [29]. This was based on a normalised cDNA library constructed from skin biopsy samples of twelve individuals and subjected to a full run on a Roche Genome Sequencer FLX Titanium, which yielded 1,443,397 454 reads of mean length $286 \mathrm{bp}$.

\section{Sequence assembly}

The pooled necropsy data and the previously sequenced skin biopsy data were separately assembled using Newbler (version 2.5.3), with a large genome style assembly followed by CAP 3 using default parameters [33] to reduce redundancy. A combined assembly using all the 454 data from both sets was then constructed in the same way to generate the best possible reference transcriptome for further analysis. After annotation using the Genbank non-redundant (nr) [41], swissprot [42] and dog transcript (ftp://ftp.ensembl.org/pub/release-67/ fasta/canis_familiaris/ref) databases, we discarded all contigs less than 500bp in length that failed to reveal homology at an e-value less than $1 \mathrm{e}^{-10}$ in at least one of these databases.

\section{BLAST mapping and sequence annotation}

To determine homology to known genes, Basic Local Alignment Search Tool (BLAST) searches [43] with a standard e-value cutoff of $1 \mathrm{e}^{-10}$ were used to query contig sequences against the GenBank nr and Swissprot databases. Gene Ontology (GO) mappings were determined using an in-house database taking the top five Swissprot matches. Immune and apoptosis related GO terms were then used to select fur seal transcripts with such matches.

\section{Comparative genomics with the dog}

Using the combined assembly, four approaches were employed to explore sequence homology between the fur seal and dog. First, individual 454 reads were mapped to the dog genome (build 2.0) using Roche gsMapper version 2.3. The dog genome sequences in fasta format were obtained from ftp://ftp.ncbi.nih.gov/ genomes/Canis_familiaris and comprised chromosomes 1 to 38 and X. Secondly, BLAST searches were carried out with the fur seal transcripts against each of the dog genome chromosomes. The highest score for a given transcript determined its placement along a chromosome. This provided the basis for positioning microsatellite and SNP loci (see below for marker discovery). Thirdly, BLAST searches were conducted comparing the assembled fur seal contigs against the set of canine nucleotide and peptide gene sequences, both annotated and abinitio, with an e-value cutoff of $1 \mathrm{e}^{-10}$.

We next compared Canis familiaris MHC genes for representation to the fur seal transcripts. These comprised 5, 12 and 4 class I, II, and III genes respectively (see Table 3 for details), identified from the literature $[44,45]$ and through searches of the dog genome at Ensembl (www.ensembl.com). Newbler was used to map the 454 reads from the separate tissues against the selected MHC genes, including the associated variant transcripts. Following Ekblom et al. [35], we then used the number of transcripts mapping per million as a proxy for gene expression.

\section{Mining for molecular markers}

The combined assembly was interrogated for microsatellite motifs using the program Phobos [46] to identify sequences containing perfect di-, tri- and tetranucleotide repeats with a minimum length of five repeat units. SNP detection was carried out using two programs. First, to provide a direct comparison with the numbers of SNPs previously obtained from the skin transcriptome [29], we applied the Swap454 pipeline [47]. This program first 
maps the raw reads back to the assembled contigs and then determines, while taking into account an error model for the 454 data, which positions are called as SNPs according to two user-specified thresholds. The first of these, 'MIN_RATIO' corresponds to the percentage of reads that differ from the reference sequence at a given position and the second, 'MIN_READS' to the number of copies present of the minor allele. We applied a 'strict' criterion to minimize the possibility of false positives arising from sequencing error and a 'relaxed' criterion to maximize the discovery of relatively infrequent alleles. For the former, MIN_RATIO was set to 0.33 and MIN_READS to 8, and for the latter MIN_RATIO was set to 0.1 and MIN_READS to 2. For comparison, we also used the Newbler mapping program (454 Life Sciences; www.454. com) to call SNPs that were deemed high confidence. For a SNP to be called in this way, there must be at least three non-duplicate reads showing the variant, with these reads being represented in both the forward and reverse directions, and at least seven reads with Phred quality scores of at least 20 .

\section{Availability of supporting data}

DNA sequences are available at the Genbank Sequence Read Archive (number: SRA064103).

\section{Additional files}

Additional file 1: Table S1. List of immune-related transcripts identified in the combined assembly with corresponding BLAST hits against the $\mathrm{nr}$ database.

Additional file 2: Table S2. The ten most commonly expressed sequences (in order of abundance) with associated BLAST matches. Data are presented sequentially for each type of tissue. Contig names correspond to the tissue-specific assemblies. Immune-related transcripts are denoted by an 'X'.

\section{Competing interests}

The authors declare that they have no competing interests.

\section{Authors' contributions}

$\mathrm{JH}$ conceived and developed the project, prepared the RNA extracts, organised the sequencing, participated in data analysis and interpretation, and wrote the manuscript. MAST conducted the bioinformatics, participated in data analysis and interpretation, and contributed to manuscript preparation. JF conducted the field monitoring and necropsies of specimens and contributed tissue samples, and JH, JF and PNT contributed funding towards the RNA extraction, CDNA synthesis and 454 sequencing. All authors commented upon and approved the final manuscript.

\section{Acknowledgements}

We would like to acknowledge library preparation and 454 sequencing performed by Shilo Dickens of the DNA sequencing facility of the Department of Biochemistry, University of Cambridge. We are also grateful to Juliane Zelwies for assistance preparing Figure 6. Fieldwork was approved by BAS and samples were collected and retained under permits issued by the Department for Environment, Food and Rural Affairs (DEFRA) and in accordance with the Convention on International Trade in Endangered Species of Wild Fauna and Flora (CITES). This work contributes to the British Antarctic Survey (BAS) Ecosystems (Polar Science for Planet Earth) programme. The research was supported by a Marie Curie FP7-Reintegration-
Grant (PCIG-GA-2011-303618) within the 7th European Community Framework Programme, together with NERC core funding to the British Antarctic Survey Ecosystems programme. We also acknowledge support of the publication fee by the Deutsche Forschungsgemeinschaft and the Open Access Publication Funds of Bielefeld University.

\section{Author details}

'Department of Animal Behaviour, University of Bielefeld, Postfach 100131, Bielefeld 33501, Germany. ${ }^{2}$ British Antarctic Survey, Natural Environment Research Council, High Cross, Madingley Road, Cambridge CB3 OET, United Kingdom.

Received: 31 October 2012 Accepted: 23 January 2013

Published: 24 January 2013

\section{References}

1. Vera JC, Wheat CW, Fescemyer HW, Frilander MJ, Crawford DL, Hanski I, Marden JH: Rapid transcriptome characterization for a non-model organism using 454 pyrosequencing. Mol Ecol 2008, 17:1636-1647.

2. Nagaraj SH, Gasser RB, Ranganathan S: A hitchhiker's guide to expressed sequence tag (EST) analysis. Briefings in Bioinformatics 2007, 8:6-21.

3. Bouck A, Vision T: The molecular ecologist's guide to expressed sequence tags. Mol Ecol 2007, 16:907-924.

4. Hudson ME: Sequencing breakthroughs for genomic ecology and evolutionary biology. Mol Ecol Resour 2008, 8:3-17.

5. Wheat CW: Rapidly developing functional genomics in ecological model systems via 454 transcriptome sequencing. Genetica 2010, 138:433-451.

6. Slate J, Santure AW, Feulner PGD, Brown EA, Ball AD, Johnston SE, Gratten J: Genome mapping in intensively studied wild vertebrate populations. Trends in Genetics 2010, 26:275-284.

7. Ouborg NJ, Pertoldi C, Loeschcke V, Bilksma R, Hedrick PW: Conservation genetics in transition to conservation genomics. Trends in Genetics 2010, 26:177-187.

8. Wang B, Ekblom R, Castoe TA, Jones EP, Kozma R, Bongcam-Rudloff E, Pollock DD, Hoglund J: Transcriptome sequencing of black grouse (Tetrao tetrix) for immune gene discovery and microsatellite development. Open Biology 2012, 2:120054.

9. Vogel H, Altincicek B, Glockner G, Vilcinkas A: A comprehensive transcriptome and immune-gene repertoire of the lepidopteran model host Galleria mellonella. BMC Genomics 2011, 12:308.

10. Clark MS, Thorne MAS, Vieira FA, Cardoso JCR, Power DM, Peck LS: Insights into shell deposition in the Antarctic bivalve Laternula elliptica: gene discovery in the mantle transcriptome using 454 pyrosequencing. BMC Genomics 2010, 11:362.

11. Renaut S, Nolte AW, Bernatchez L: Mining transcriptome sequences towards identifying adaptive single nucleotide polymorphisms in lake whitefish species pairs (Coregonus spp. Salmonidae). Mol Ecol 2010, 19:115-131.

12. Kang HJ, Kawasawa YI, Cheng F, Zhu Y, Xu X, Li M, Sousa AM, Pletikos M, Meyer KA, Sedmak G, et al: Spatio-temporal transcriptome of the human brain. Nature 2011, 478:483-489.

13. Cherel P, Herault F, Vincent A, Le Roy P, Damon M: Genetic variability of transcript abundance in pig skeletal muscle at slaughter: relationships with meat quality traits. J Anim Sci 2011, 90:699-708.

14. Hoffman Jl, Grant SM, Forcada J, Phillips CD: Bayesian inference of a historical genetic bottleneck in a heavily exploited marine mammal. Mol Ecol 2011, 20:3989-4008.

15. Read AJ: The looming crisis: interactions between marine mammals and fisheries. J Mammal 2008, 89:541-548.

16. Simmonds MP, Isaac SJ: The impacts of climate change on marine mammals: early signs of significant problems. Oryx 2007, 41:19-26.

17. Mancia A, Lundqvist ML, Romano TA, Peden-Adams M, Fair PA, Kindy MS, Ellis BC, Gattoni-Celli S, McKillen DJ, Trent HF, et al: A dolphin peripheral blood leukocyte CDNA microarray for studies of immune function and stress reactions. Dev Comp Immunol 2007, 31:520-529.

18. lerardi JL, Mancia A, McMillan J, Lundqvist ML, Romano TA, Wise JP Sr, Warr GW, Chapman RW: Sampling the transcriptome of the North Atlantic right whale. Comp Biochem Physiol, Part D 2009, 4:154-158.

19. Osterhaus ADME, Groen J, Spijkers HEM, Broeders HWJ, UytdeHaag FGCM, de Vries P, Teppema JS, Visser IKG, van de Bildt MWG, Vedder EJ: Mass 
mortality in seals caused by a newly discovered virus-like morbillivirus. Vet Microbiol 1990, 23:343-350.

20. Mattlin RH: Pup mortality of the New Zealand fur seal (Arctocephalus forsteri lesson). N Z J Ecol 1978, 1:138-144.

21. Pyenson ND: The high fidelity of the cetacean stranding record: insights into measuring diversity by integrating taphonomy and macroecology. Proc $R$ Soc London, Ser B, online early.

22. McCann TS: Territoriality and breeding behaviour of adult male Antarctic fur seal, Arctocephalus gazella. J Zool (London) 1980, 192:295-310.

23. Doidge DW, Croxall JP, Ricketts C: Growth rates of Antarctic fur seal Arctocephalus gazella pups at South Georgia. J Zool (London) 1984, 203(MAY):87-93.

24. Hoffman JI, Boyd IL, Amos W: Male reproductive strategy and the importance of maternal status in the Antarctic fur seal Arctocephalus gazella. Evolution 2003, 57(8):1917-1930.

25. Hoffman JI, Boyd IL, Amos W: Exploring the relationship between parental relatedness and male reproductive success in the Antarctic fur seal Arctocephalus gazella. Evolution 2004, 58(9):2087-2099.

26. Hoffman Jl, Forcada J, Amos W: Getting long in the tooth: a strong positive correlation between canine size and heterozygosity in the Antarctic fur seal Arctocephalus gazella. J Hered 2010, 101:527-538.

27. Hoffman JI, Forcada J, Trathan PN, Amos W: Female fur seals show active choice for males that are heterozygous and unrelated. Nature 2007, 445:912-914.

28. Hoffman Jl, Forcada J, Amos W: Exploring the mechanisms underlying a heterozygosity-fitnesss correlation for canine size in the Antarctic fur seal Arctocephalus gazella. J Hered 2010, 101:539-552.

29. Hoffman Jl: Gene discovery in the Antarctic fur seal (Arctocephalus gazella) skin transcriptome. Mol Ecol Resour 2011, 11:703-710.

30. Hoffman Jl, Nichols HJ: A novel approach for mining polymorphic microsatellite markers in silico. PLoS One 2011, 6:e23283.

31. Hoffman Jl, Tucker R, Clark MS, Forcada J, Slate J: Rates of assay success and genotyping error when single nucleotide polymorphism genotyping in non-model organisms: a case study in the Antarctic fur seal. Mol Ecol Resour 2012, 12:861-872.

32. da Assunção Soares Franco M, Hoffman Jl, Harwood J, Amos W: MHC genotype is a dominant predictor of mortality in the grey seal. Scientific Reports 2012, 2:659.

33. Huang $X$, Madan A: CAP 3: a DNA sequence assembly program. Genome Res 1999, 9:868-877.

34. Kumar S, Blaxter ML: Comparing de novo assemblers for 454 transcriptome data. BMC Genomics 2010, 11:571.

35. Ekblom R, Balakrishnan CN, Burke T, Slate J: Digital gene expression analysis of the zebra finch genome. BMC Genomics 2010, 11:219.

36. Santure AW, Gratten J, Mossman JA, Sheldon BC, Slate J: Characterisation of the transcriptome of a wild great tit Parus major population by next generation sequencing. BMC Genomics 2011, 12:283.

37. Baker JR, Doidge DW: Pathology of the Antarctic fur seal (Arctocephalus gazella) in South Georgia. Br Vet J 1984, 140:210-219.

38. Acevedo-Whitehouse $K$, Cunningham AA: Is MHC enough for understanding wildlife immunogenetics? Trends Ecol Evol 2006, 21(8):433-438.

39. Higdon JW, Bininda-Edmonds ORP, Beck RMD, Ferguson SH: Phylogeny and divergence of the pinnipeds (Carnivora: Mammalia) assessed using a multigene dataset. BMC Evol Biol 2007, 7:216.

40. Osborne AJ, Brauning R, Schultz JK, Kennedy MA, Slate J, Gemmell NJ: Development of a predicted physical map of microsatellite locus positions for pinnipeds, with wider applicability to the Carnivora. Mol Ecol Resour 2010, 11:503-513.

41. Galperin MY, Cochrane GR: The 2011 nucleic acids research database issue and the online molecular biology database collection. Nucleic Acids Res 2011, 39:D1-D6.

42. Bairoch A, Apweiler R: The SWISS-PROT protein sequence data bank and its new supplement TREMBL. Nucleic Acids Res 1996, 24:21-25.

43. Altschul SF, Gish W, Miller W, Myers EW, L DJ: Basic local alignment search tool. J Mol Biol 1990, 215:403-410.

44. Wagner JL: Molecular organization of the canine major histocompatibility complex. J Hered 2003, 94(1):23-26.
45. Debenham SL, Hart EA, Ashurst JL, Howe KL, Quail MA, Ollier WER, Binns $\mathrm{MM}$ : Genomic sequence of the class I region of the canine MHC: comparison with the MHC of other mammalian species. Genomics 2005, 85:48-59.

46. Phobos 3.3.11. http://www.rub.de/spezzoo/cm/cm_phobos.htm.

47. Brockman W, Alvarez P, Young S, Garber M, Giannoukos G, Lee WL, Russ C, Lander ES, Nusbaum C, Jaffe DB: Quality scores and SNP detection in sequencing-by-synthesis systems. Genome Res 2008, 18:763-770.

doi:10.1186/1471-2164-14-52

Cite this article as: Hoffman et al:: Transcriptome of the dead: characterisation of immune genes and marker development from necropsy samples in a free-ranging marine mammal. BMC Genomics 2013 14:52.

\section{Submit your next manuscript to BioMed Central and take full advantage of:}

- Convenient online submission

- Thorough peer review

- No space constraints or color figure charges

- Immediate publication on acceptance

- Inclusion in PubMed, CAS, Scopus and Google Scholar

- Research which is freely available for redistribution 